\title{
Long-chain polyunsaturated fatty acids attenuate the IL-1 $\beta$-induced proinflammatory response in human fetal intestinal epithelial cells
}

\author{
Vasuki Wijendran' ', J.T. Brenna², Dong Hao Wang ${ }^{2}$, Weishu Zhu', Di Meng', Kriston Ganguli', Kumar S.D. Kothapalli², \\ Pilar Requena ${ }^{3}$, Sheila Innis ${ }^{4}$ and W. Allan Walker ${ }^{1}$
}

BACKGROUND: Evidence suggests that excessive inflammation of the immature intestine may predispose premature infants to necrotizing enterocolitis (NEC). We investigated the anti-inflammatory effects of docosahexaenoic acid (DHA) eicosapentaenoic acid (EPA), and arachidonic acid (ARA) in human fetal and adult intestinal epithelial cells (IEC) in primary culture.

METHODS: Human fetal IEC in culture were derived from a healthy fetal small intestine $(\mathrm{H} 4)$ or resected small intestine of a neonate with NEC (NEC-IEC). Intestinal cell lines Caco2 and NCM460 in culture were used as models for mature IEC. IEC in culture were pretreated with $100 \mathrm{\mu mol} / \mathrm{l}$ palmitic acid (PAL), DHA, EPA, ARA, or ARA+DHA for $48 \mathrm{~h}$ and then stimulated with proinflammatory IL-1 $\beta$.

RESULTS: DHA significantly attenuated IL-1 $\beta$ induced proinflammatory IL-8 and IL-6 protein and mRNA in fetal H4, NECIEC, and mature Caco2, NCM460 IEC, compared to control and PAL treatment. DHA downregulated IL-1R1 (IL-1 $\beta$ receptor) and NFk $\beta 1$ mRNA expression in fetal and adult IEC. ARA had potent anti-inflammatory effects with lower IL-8 and IL-6 (protein and mRNA) in fetal $\mathrm{H} 4$ but not in NEC-IEC or adult IEC.

CONCLUSION: The present study provides evidence that DHA and ARA may have important anti-inflammatory functions for prevention of NEC in premature infants.

N ecrotizing enterocolitis (NEC) is the most devastating gastrointestinal disease in neonates, especially affecting verylow-birth-weight premature infants (1). The pathogenesis of NEC is not well defined but evidence strongly suggests that it is multifactorial $(2,3)$. Prematurity and enteral feeding are major risk factors for NEC $(1,2)$. An excessive inflammatory response by the immature intestine to external stimuli, impaired intestinal barrier integrity and/or abnormal bacterial colonization are key factors implicated in the pathophysiology of NEC (1-3). Current evidence strongly suggest that immaturity of innate immunity mediated via TLR4 and NFk $\beta 1$ signaling pathway may contribute to excessive intestinal inflammation in NEC (4-6).
Breast milk protects the immature intestine against excessive inflammation underlying NEC $(1,3)$. The long chain polyunsaturated fatty acids (LC-PUFA, $\geq 20$ carbon PUFA) docosahexaenoic acid (DHA, 22:6n-3) and arachidonic acid (ARA, 20:4n-6) present in human breast milk (7) may have important anti-inflammatory functions during early development $(8,9)$. A recent meta-analysis study of randomized clinical trials reported that DHA supplementation reduced the risk of NEC in premature infants with gestational age $\leq 32 \mathrm{wk}$ (8). Carlson et al. (9) also reported a decrease in the incidence of NEC in premature infants fed a preterm formula supplemented with DHA and ARA compared to a standard formula without DHA and ARA. However, Fewtrell et al. (10) did not find any effect of DHA and ARA supplementation on the incidence of NEC in premature infants. Further, the mechanisms underlying the anti-inflammatory functions of DHA and ARA in immature intestine during early fetal development are currently not well defined.

Accordingly, the primary goal of the present study was to determine the ant-inflammatory effects of DHA, eicosapentaenoic acid (EPA, 20:5n-3) and ARA and delineate their mechanisms of action in human fetal intestinal epithelial cells (IEC) in primary culture as a model for fetal (immature) human intestine. The specific objectives of the study were to determine the effects of DHA, EPA, and ARA, within physiological ranges present in human breast milk (7), on (i) the proinflammatory cytokines IL- 8 and IL-6 response and (ii) $\mathrm{NFk} \beta 1$ and IL1-R1 gene expression in human fetal and adult IEC in culture, after proinflammatory IL-1 $\beta$ insult.

\section{RESULTS}

Fatty Acid Enrichment of Human Fetal (H4, NEC-IEC) and Adult (Caco2) Intestinal Epithelial Cells in Culture After Supplementation

Fatty acid enrichment measured in human fetal H4 IEC, NECIEC, and adult Caco 2 after $48 \mathrm{~h}$ of supplementation with palmitic acid (PAL), ARA, EPA, DHA and ARA+DHA, is shown in Table 1. All fatty acids supplemented showed enrichment in 
Table 1. Fatty acid enrichment in $\mathrm{H} 4, \mathrm{NEC}$, and $\mathrm{CaCO} 2 \mathrm{IEC}$ in culture after supplementation

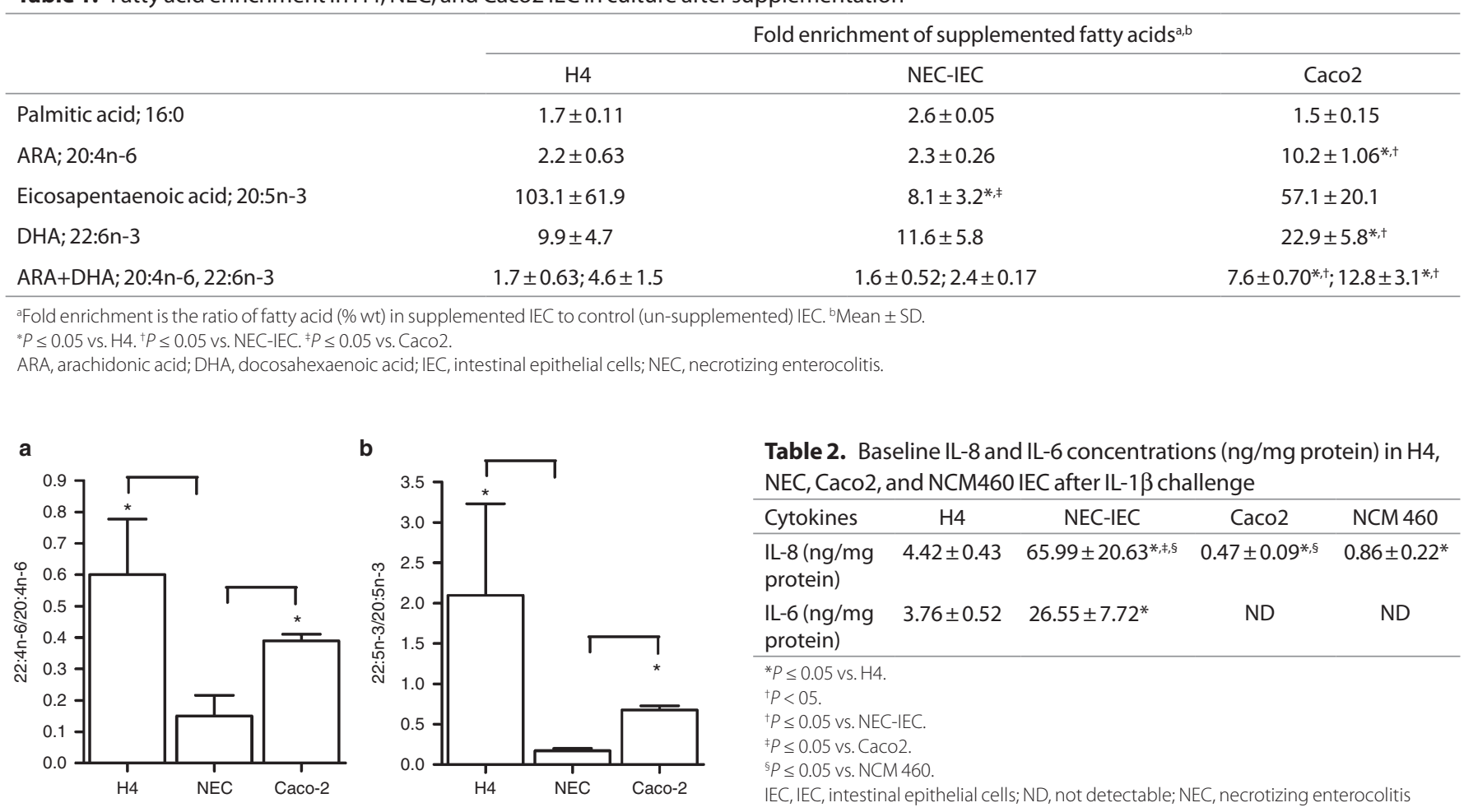

Figure 1. Elongation of arachidonic acid (ARA) and eicosapentaenoic acid (EPA) to 22 carbon products in human fetal $\mathrm{H} 4$, necrotizing enterocolitis (NEC) and adult Caco2 IEC. (a) 22:4n-6/20:4n-6 ratio in H4, NEC, and Caco2 IEC after ARA supplementation and (b) 22:5n-3/20:5n-3 ratio in H4, NEC, and Caco2 IEC after EPA supplementation. ${ }^{*} P \leq 0.05$.

H4, NEC-IEC, and Caco2 IEC in culture. In fetal IEC, DHA increased by 10 - and 12 -fold in $\mathrm{H} 4$ and NEC-IEC respectively compared to unsupplemented control IEC. EPA was 103-fold higher in H4 IEC, but only eightfold higher in NEC-IEC. ARA increased by twofold in $\mathrm{H} 4$ and NEC-IEC. In adult Caco2, ARA, EPA, and DHA increased by 10-, 57-, and 23-fold. The fatty acid composition (\% total fatty acids) of fetal and adult IEC in culture is shown in Supplementary Table S1 online. ARA supplementation significantly lowered DHA in IEC. Similarly, DHA supplementation led to a significant decrease in ARA in IEC.

Significant 2 carbon elongation of 20-carbon PUFA ARA and EPA (as indicated by $22: 4 n-6 / 20: 4 n-6$ and $22: 5 n-3 / 20: 5 n-3$ ratios, respectively) was evident in fetal H4 IEC (Figure 1). In contrast, in NEC-IEC, 22:4n-6/20:4n-6 ratio and 22:5n-3/20:5n-3 ratios were 2- and 15 -fold lower respectively compared to H4 IEC.

ARA or EPA supplementation did not increase the levels of their down-stream desaturation products namely, 22:5n-6 or DHA in fetal (H4, NEC) or adult (Caco2) IEC, indicating a low fatty acid desaturase 2 (FADS2) activity in IEC.

\section{Fatty Acid Effects on Proinflammatory Cytokine Response After IL-1 $\beta$ Challenge in Human Fetal ( $\mathrm{H} 4$ and NEC) and Adult (Caco2 and NCM460) Intestinal Epithelial Cells}

IL-8 and IL-6 concentration (ng/mg protein) in cell-fee supernatant (CFS) of unsupplemented (control) fetal and adult IEC after IL- $1 \beta$ exposure is shown in Table 2 . IL- 8 and IL-6 protein concentration (ng/mg protein) was highest in fetal NECIEC, followed by $\mathrm{H} 4$ and lowest in adult Caco2 and NCM400 enterocytes (Table 2). In NEC-IEC, IL-8 and IL-6 concentrations (ng/mg) in CFS after IL-1 $\beta$ exposure were 14 - and 7-fold greater compared to H4 IEC. IL-6 concentration was not detectable in adult Caco 2 or NCM CFS after IL- $1 \beta$ challenge.

IL-8 protein (ng/mg protein, Figure 2) and its mRNA expression (Figure 3 ) was measured in fetal and adult IEC supplemented with PAL, ARA, EPA, DHA, or ARA+DHA compared to control IEC after IL- $1 \beta$ exposure. DHA significantly attenuated the IL- 8 protein response to IL- $1 \beta$ in fetal H4, NEC-IEC and adult Caco2, NCM460 IEC compared to control and PAL treated IEC. In NEC-IEC, DHA decreased IL- 8 levels by $41 \%$ compared to control after IL- $1 \beta$ challenge. IL- 8 mRNA expression was also significantly lower in DHA treated fetal $\mathrm{H} 4$ and adult Caco 2 cells compared to control and PAL. EPA significantly decreased the IL-8 response to IL- $1 \beta$ in H4 IEC, but not in NEC-IEC or adult Caco2 or NCM460 IEC. ARA had potent anti-inflammatory effects on fetal H4 IEC with significantly lower IL-8 protein and mRNA compared to control and PAL treated IEC. ARA decreased IL-8 levels in H4 IEC by $58 \%$ compared to control. However, ARA did not attenuate pro-inflammatory IL-8 response to IL-1 $\beta$ in NEC-IEC or in Caco 2 or NCM460 IEC. ARA+DHA decreased IL-8 response to IL-1 $\beta$ in fetal H4 IEC compared to control and PAL.

LC-PUFA effects on proinflammatory IL-6 protein concentration (ng/mg protein) (Figure 4) and mRNA expression (Figure 5) in fetal H4 and NEC-IEC were similar to their effects on the IL-8 response. DHA significantly decreased IL-6 


\section{Articles | Wijendran et al.}

a

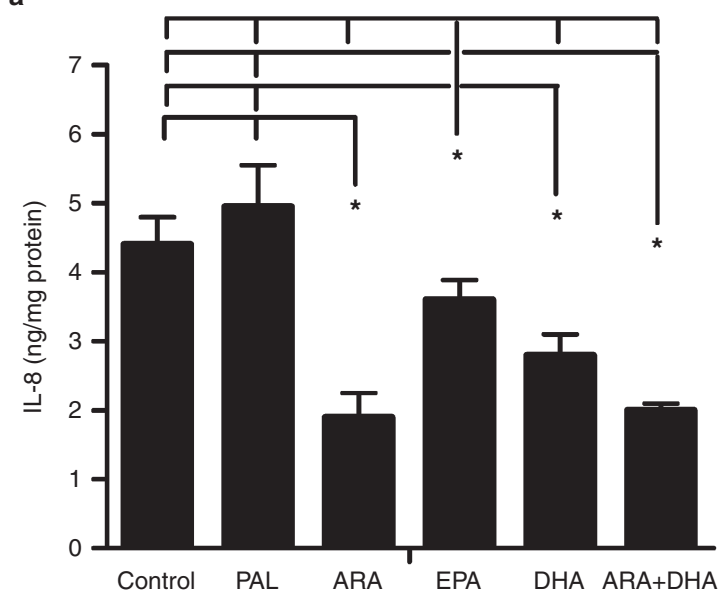

c

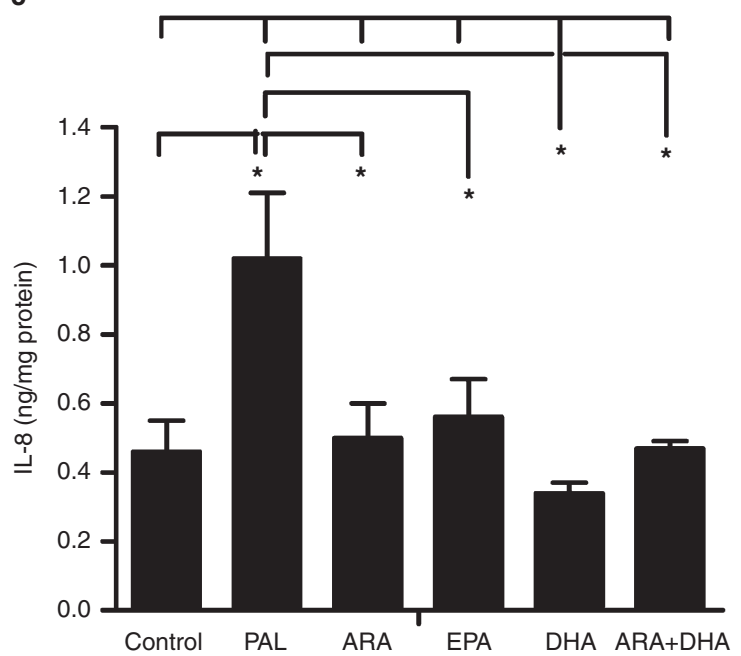

b
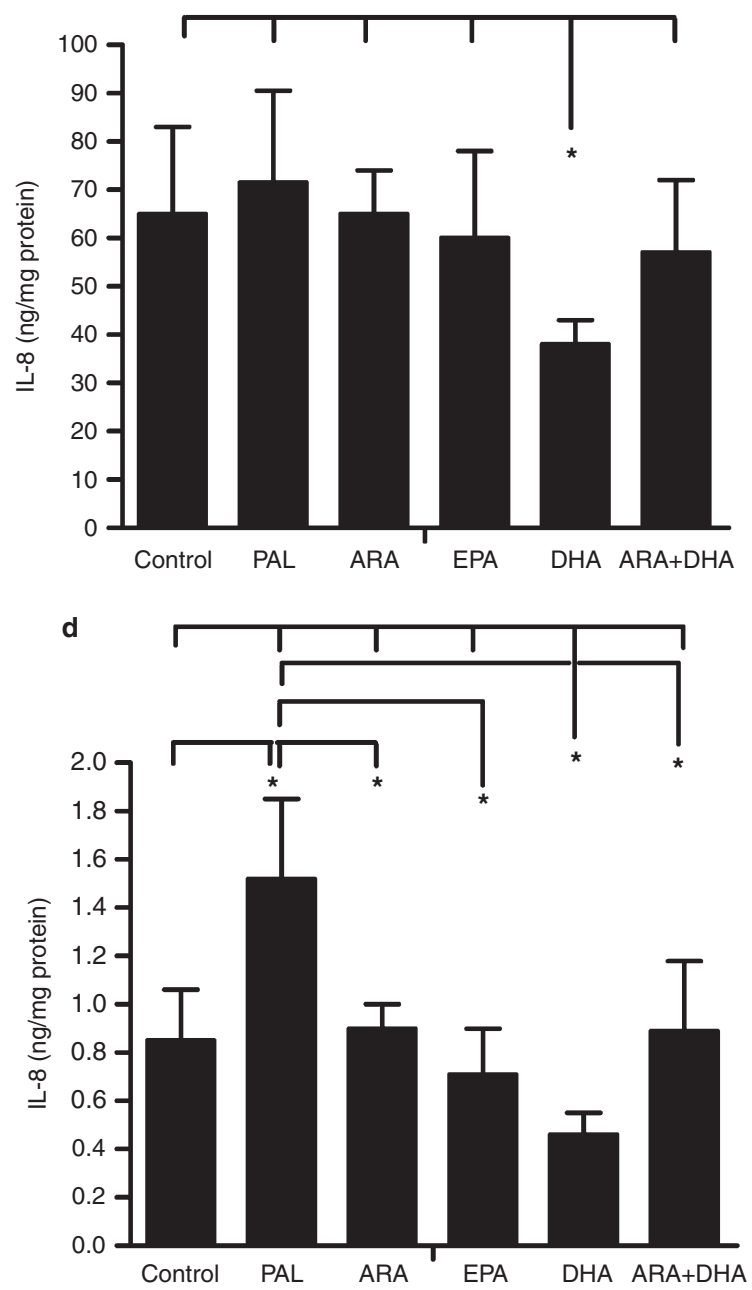

Figure 2. IL-8 protein concentration (ng/mg protein) in cell-free supernatant of human (a) fetal H4 necrotizing enterocolitis (IEC), (b) NEC-IEC, (c) adult Caco2, and (d) adult NCM 460 IEC after fatty acid supplementation and IL- $1 \beta$ stimulation. (a) $* P \leq 0.05$, arachidonic acid (ARA) vs., control, palmitic acid (PAL); eicosapentaenoic acid (EPA) vs. all other groups; docosahexaenoic acid (DHA) vs. control, PAL, ARA+DHA vs. control and PAL; (b) *P $\leq 0.05, \mathrm{DHA}$ vs. all other groups; $(\mathbf{c}) * P \leq 0.05$ PAL vs. control and ARA; EPA vs. PAL; DHA vs. all other groups, ARA+DHA vs. PAL.

a

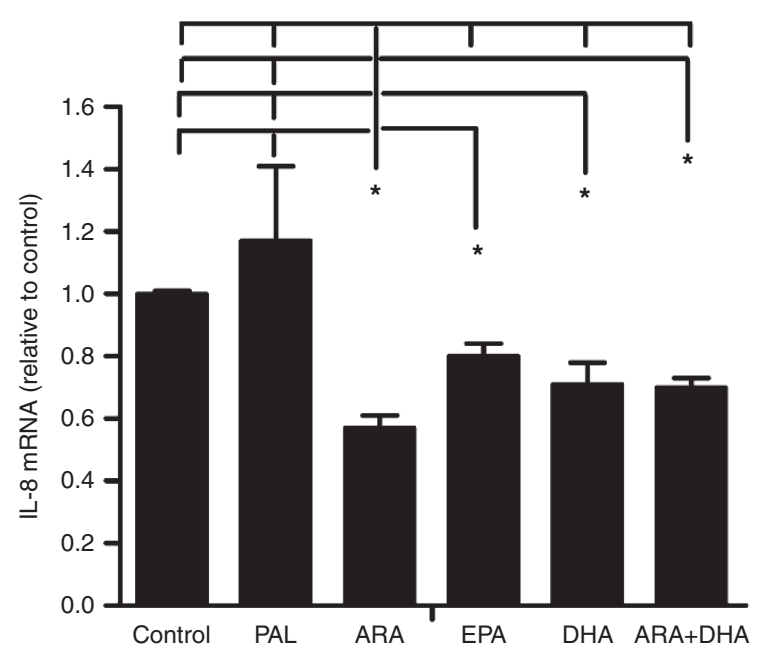

b

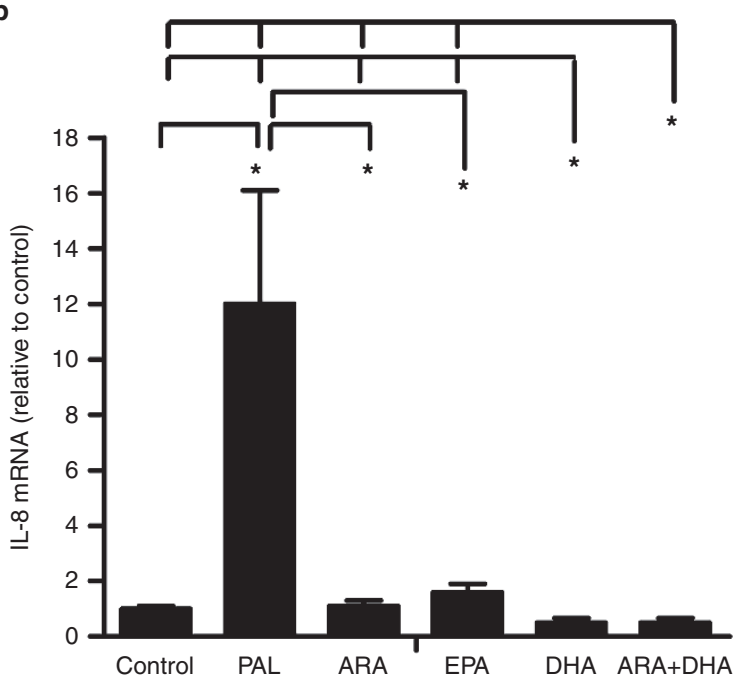

Figure 3. IL-8 mRNA expression (relative to control IEC arbitrarily set as 1 in human fetal $\mathrm{H} 4$ and adult Caco2 necrotizing enterocolitis (IEC)) after fatty acid supplementation and IL-1 $\beta$ stimulation. IL- 8 mRNA in (a) fetal H4 and (b) adult Caco2 IEC. ${ }^{*} P \leq 0.05$; (a) fetal H4 IEC, ARA vs. all other groups; eicosapentaenoic acid (EPA) vs. control and palmitic acid (PAL); docosahexaenoic acid (DHA) vs. control and PAL; ARA+DHA vs. control and PAL; (b) adult Caco 2 IEC, PAL vs. control; arachidonic acid (ARA) vs. PAL; EPA vs. PAL; DHA vs. all other groups except ARA+DHA. 
a

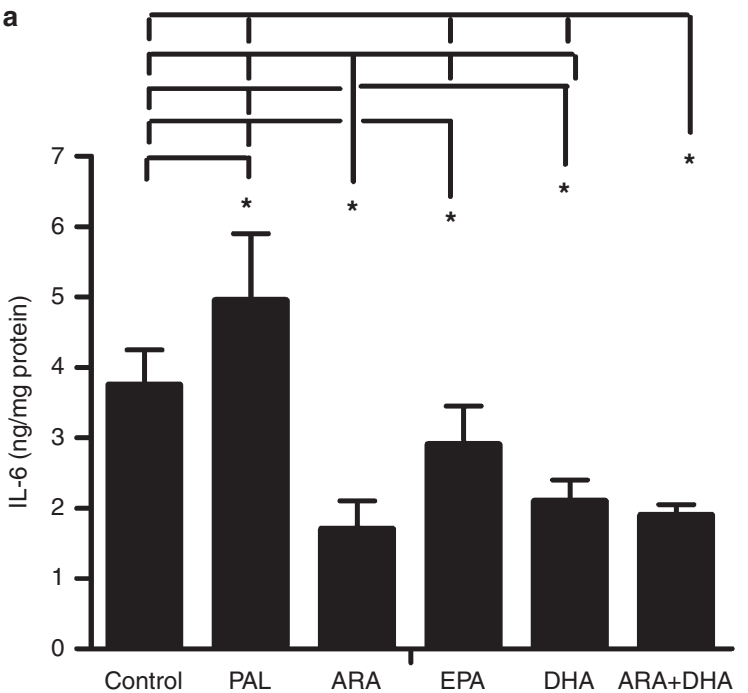

b

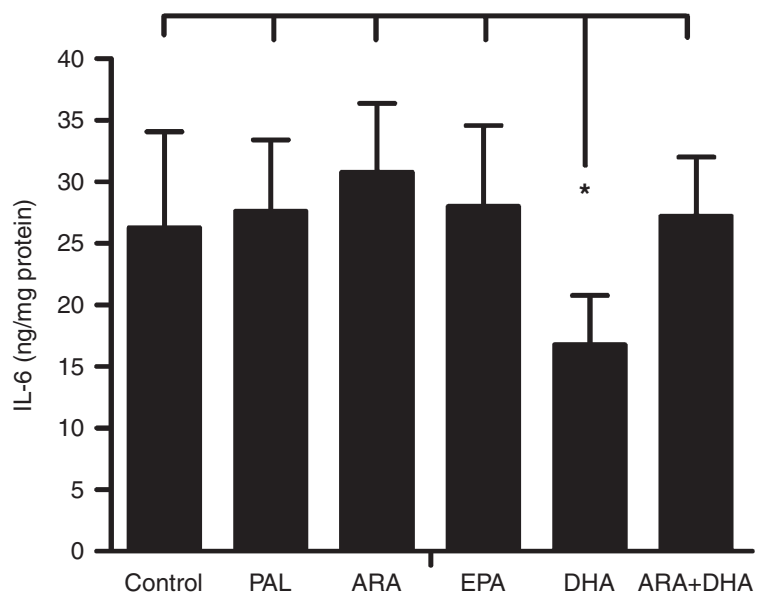

Figure 4. IL-6 protein concentration ( $\mathrm{ng} / \mathrm{mg}$ protein) in cell-free supernatant of human fetal $\mathrm{H} 4$ and NEC-IEC after fatty acid supplementation and IL-1 $\beta$ stimulation. IL-6 (ng/mg protein) in (a) fetal $\mathrm{H} 4$ and (b) NEC-IEC. ${ }^{*} P \leq 0.05$; (a) fetal H4IEC, palmitic acid (PAL) vs. control; ARA vs. all other groups except ARA+DHA; eicosapentaenoic acid (EPA) vs. control and PAL; docosahexaenoic acid (DHA) vs. control and PAL; ARA+DHA vs. all other groups except arachidonic acid (ARA); b) adult Caco 2 IEC, DHA vs. all other groups.

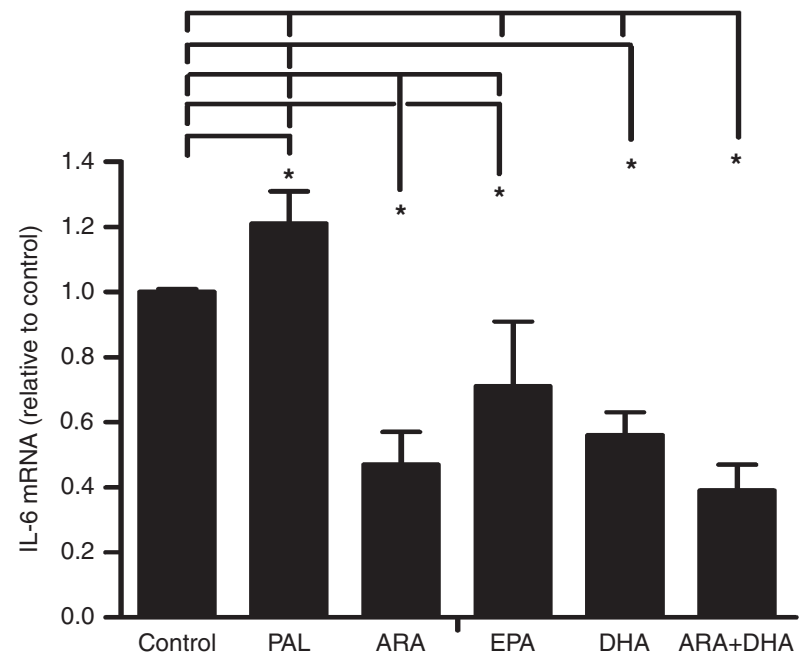

Figure 5. IL-6 mRNA expression (relative to control IEC arbitrarily set as 1 in human fetal H4 IEC) after fatty acid supplementation and IL-1 $\beta$ stimulation. ${ }^{*} P \leq 0.05$ palmitic acid (PAL) vs. control; arachidonic acid (ARA) vs. control, PAL and eicosapentaenoic acid (EPA); EPA vs. control and PAL; docosahexaenoic acid (DHA) vs. control and PAL: ARA+DHA vs. all other groups except ARA.

levels (ng/mg protein) in the CFS of $\mathrm{H} 4$ and NEC-IEC and its mRNA in H4 IEC compared to control and PAL. ARA significantly lowered the IL- 6 response (protein and mRNA) in $\mathrm{H} 4$ cells but not in NEC-IEC.

\section{Fatty Acid Effects on Gene Expression of IL-1R1 and NFk $\beta 1$ in H4 and Caco2 IEC After IL-1 $\beta$ Exposure}

Expression levels of the IL-1 $\beta$ receptor (IL-1R1) and the down-stream signaling molecule NFk $\beta 1$ mRNA were sevenand fivefold higher respectively in $\mathrm{H} 4$ cells compared to Caco2 cells (Figure 6). Relative gene expression (normalized to control unsupplemented IEC) of the IL- $1 \beta$ receptor (IL-1R1) and its down-stream proinflammatory signaling mediator $\mathrm{NFk} \beta 1$

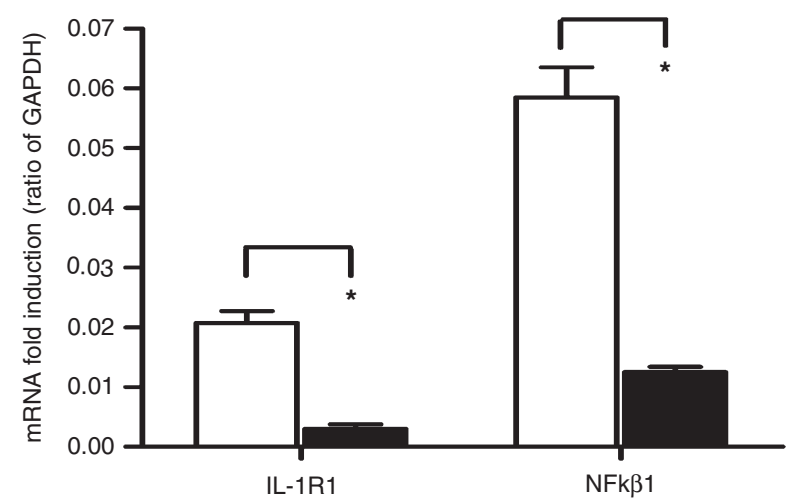

Figure 6. IL-1R1 and NFk $\beta 1 \mathrm{mRNA}$ fold induction (expressed as a ratio of house-keeping gene GAPDH) in human fetal $\mathrm{H} 4$ and adult $\mathrm{Caco} 2$ control IEC after IL-1 $\beta$ stimulation. $\square \mathrm{H} 4 \square$ Caco2. ${ }^{*} P \leq 0.05$ vs. $\mathrm{H} 4$.

in $\mathrm{H} 4$ and $\mathrm{Caco} 2$ enterocytes $4 \mathrm{~h}$ after IL- $1 \beta$ challenge is shown in Figure 7. DHA significantly lowered IL-1R1 and NFk $\beta 1$ mRNA in $\mathrm{H} 4$ and Caco2 IEC compared to control and PAL. In H4 IEC, DHA downregulated IL-1R1 and NFk $\beta 1$ by twoand 1.5-fold compared to control. ARA or EPA did not significantly alter IL-1R1 or NFk $\beta 1$ expression compared to control in $\mathrm{H} 4$ or $\mathrm{Caco} 2$ cells.

\section{DISCUSSION}

The present study provides evidence that DHA and ARA may have significant anti-inflammatory functions in the human fetal intestinal epithelium. To our knowledge, this is the first report investigating DHA, EPA and ARA effects, at a physiological range present in human breast milk, on the inflammatory response in human fetal IEC. Previous studies from our laboratory have well established the human fetal $\mathrm{H} 4$ and NEC-IEC in primary culture, used in the present study, as a valid model for immature human intestine to investigate the mechanisms underlying NEC $(4,11,12)$. 
a

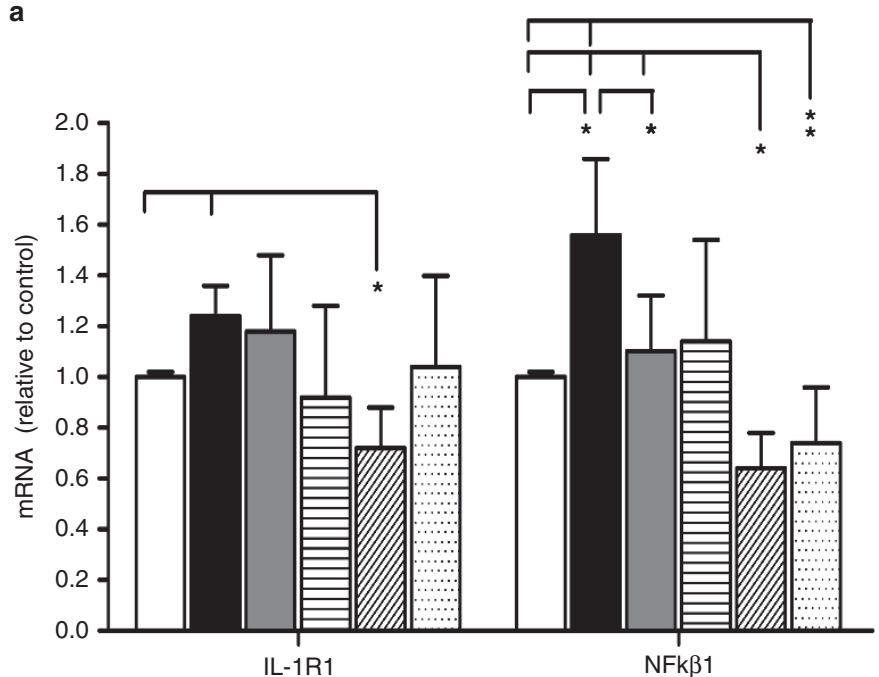

b

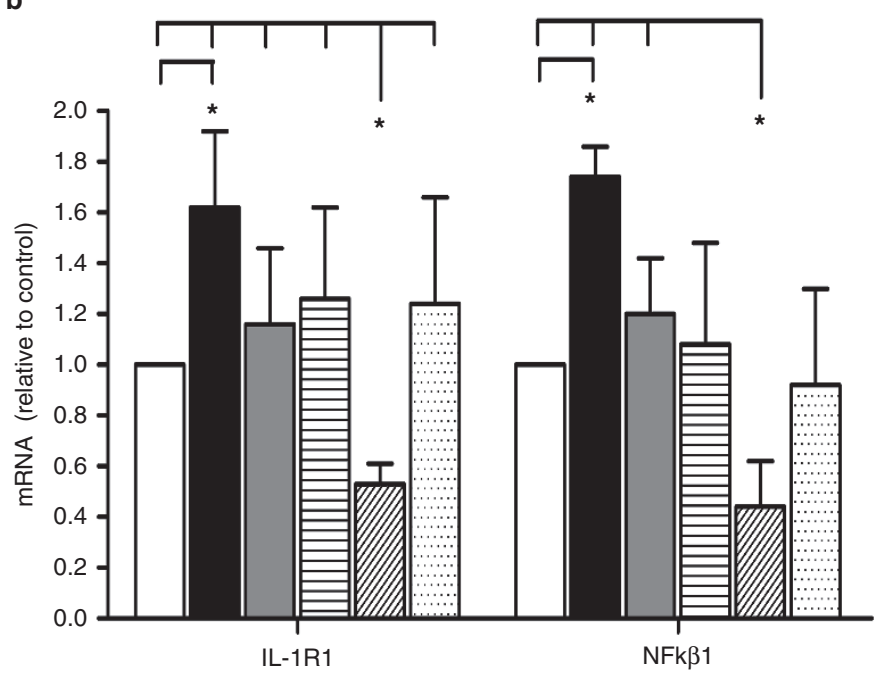

Figure 7. IL-1R1 and NFk $\beta 1$ mRNA expression (relative to control IEC arbitrarily set as 1) in (a) human fetal H4 and (b) adult Caco2 IEC after fatty acid

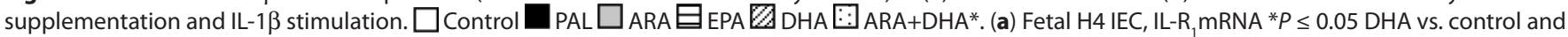
palmitic acid (PAL); NFKB1 mRNA; *P 0.05 PAL vs. control; ARA vs. PAL; DHA vs. control, PAL and arachidonic acid (ARA); ARA+DAH vs. control and PAL. (b) Fetal H4 IEC. IL-R mRNA, * $\mathrm{p} \leq 0.05 \mathrm{PAL}$ vs. control; DHA vs. all other groups. Fetal H4 IEC NFKB1 mRNA, *P $\leq 0.05 \mathrm{PAL}$ vs. control; DHA vs. control, PAL, and ARA.

Results from the current study demonstrate a significant enrichment of human fetal and adult IEC with DHA, EPA, and ARA, $48 \mathrm{~h}$ after supplementation of the respective LC-PUFA. These findings are consistent with previous in vivo animal studies $(13,14)$. Fetal and adult IEC showed preferential enrichment of n-3 LC-PUFA EPA and DHA. Further, the present study provides evidence for significant elongation of EPA and ARA to 22 carbon LC-PUFA in healthy human fetal H4 IEC. Significant 22 carbon LC-PUFA enrichment in H4 IEC supplemented with EPA and ARA suggests a relatively high activity of elongases involved in the elongation of n-3 and n-6 LC-PUFA, such as Elovl2, Elovl4, and/or Elovl5, in human fetal intestinal epithelial cells. The 22 carbon LC-PUFA and their very long chain PUFA (VLC-PUFA, $\geq 24$ carbon fatty acids) derivatives are essential components of membrane lipids $(15,16)$ and may enhance intestinal barrier integrity. Preliminary data from this study suggest that elongation of EPA and ARA to 22 carbon LC-PUFA may be impaired in NEC-IEC compared to healthy fetal H4 IEC. Impairment in elongation of precursors EPA and ARA observed in NEC IEC may alter downstream VLC-PUFA synthesis and potentially contribute to intestinal barrier dysfunction. These are preliminary observations and only suggest that failure of PUFA elongation in NEC-IEC may contribute to NEC inflammation indirectly through barrier dysfunction. Additional in-depth dose-response studies are needed before specific conclusions can be drawn. Future in vivo animal and clinical studies are needed to determine the functional role of intestinal elongases involved in the elongation of n-3 and n-6 LC-PUFA and their clinical significance in the pathogenesis of NEC.

DHA supplementation, at a physiological concentration present in human breast milk $(100 \mu \mathrm{mol} / \mathrm{l})$, decreased proinflammatory IL-8 and IL-6 secretion (ng/mg protein) and their mRNA expression in both fetal and adult human IEC, after IL-1 $\beta$ challenge. A combination of DHA with ARA (ARA+DHA at $50 \mu \mathrm{mol} / \mathrm{l}$ concentration each) also lowered the IL-8 and IL-6 response in immature human enterocytes, suggesting a potentially increased effect in immature enterocytes. It is interesting to note that anti-inflammatory effects of DHA were more pronounced in human NEC-IEC compared to H4 IEC and mature enterocytes (Caco2, NCM 460), suggesting a functionally significant role for DHA in attenuating severity of inflammation in immature intestine and possibly in attenuating NEC. Previous human clinical $(8,9)$ and in vivo animal $(13,17,18)$ studies have shown DHA to attenuate immature intestinal inflammation and lower the incidence of NEC. Findings from this study confirm that DHA has direct antiinflammatory effects on human fetal and adult intestinal epithelial cells, but a greater effect in the fetal enterocytes, again supporting the potential preventative effect of DHA with NEC.

An interesting finding on the mechanism underlying the observed anti-inflammatory effects of DHA is that DHA decreased the IL-1R1 receptor expression and the downstream proinflammatory signaling mediator $\mathrm{NFk} \beta 1$ gene expression in fetal and adult human IEC compared to control and PAL supplemented IEC. IL-1R1 and NFk $\beta 1$ expression after IL- $1 \beta$ exposure in fetal H4 IEC were five- to sevenfold higher than their expression in adult Caco2 IEC, indicating exaggerated innate immune inflammatory response in human fetal IEC. IL-1R1, the trans-membrane receptor for IL- $1 \beta$, is similar to toll like receptors (TLR4) in activating NFk $\beta 1$ signaling pathway involved in innate immunity (19). Current evidence strongly suggest that an exaggerated TLR4 and NFk $\beta 1$ expression in immature and NEC intestine may be a major factor underlying the excessive proinflammatory response in $\operatorname{NEC}(4,6,20)$. Further, a recent report on genome-wide analysis of human NEC intestinal tissues identified aberrations in the NFk $\beta 1$ signaling pathway as one possible major functional 
pathway contributing to the pathogenesis of NEC (5). The present study provides evidence that in human immature and mature intestinal epithelial cells, DHA attenuated inflammation by a downregulation of proinflammatory $N F k \beta 1$ gene expression. In vivo animal studies have shown that DHA and/ or fish oil containing DHA downregulated the intestinal TLR4 and NFk $\beta 1$ signaling pathway in rat models of $\operatorname{NEC}(17,18)$ and neonatal piglets after LPS challenge (13). Thus, the mechanism underlying anti-inflammatory effects of DHA in fetal and adult intestinal inflammation may in part involve the common axis of downregulating NFk $\beta 1$-mediated innate immunity. Others have shown that DHA/PUFAs can modulate other inflammatory pathways $(8,18,21)$ suggesting that DHA/PUFAs affect multiple pathways in intestinal inflammation. We plan transcription profiling of $\mathrm{H} 4$ and NEC-IEC cells exposed to DHA and IL- $1 \beta$ to determine the extent of these pathways.

The n-6 LC-PUFA ARA had potent anti-inflammatory effects in healthy fetal H4 IEC but not in NEC-IEC or adult Caco2 or NCM460 IEC. Further, ARA did not modulate the IL1-R1 or NFk $\beta 1$ gene expression in fetal or adult enterocytes. Thus, ARA and DHA likely modulate fetal intestinal inflammation via different mechanisms. Additional cellular studies are needed to determine these mechanisms. Recent evidence demonstrates that ARA and its eicosanoid derivatives are essential for the enhanced structural and functional integrity of intestinal barrier during early development $(14,22)$. Innis et al. (14) reported that supplementation of the maternal diet with ARA during early gestation led to enrichment of ARA in fetal colon epithelium and protected the offspring against adult onset colitis, suggesting programming of intestinal barrier integrity by ARA during early development. Our finding that ARA supplementation attenuated the proinflammatory IL-8 and IL-6 response to IL- $1 \beta$ stimulus in human immature H4 IEC only, but not in adult Caco2 or NCM460 IEC, provide additional evidence for the developmental regulation of intestinal inflammation by ARA.

EPA had modest anti-inflammatory effects on healthy fetal H4 IEC, but no effects on NEC-IEC or adult IEC in culture. Significant enrichment of EPA and its elongation product 22:5n-3 in healthy fetal H4 IEC suggests that EPA, similar to ARA, may play an important role in structural and functional integrity of fetal intestinal cell membrane during early human development. A recent study (23) in human macrophage cells demonstrated that 22:5n-3 derived from EPA and 22:4 n-6 derived from ARA potently inhibited proinflammatory eicosanoid synthesis from ARA by shunting ARA to an anti-inflammatory lipoxygenase pathway. Developmental regulation of ARA and EPA metabolism to shunt these eicosanoid precursors toward the anti-inflammatory lipoxygenase pathway and/or to utilize these LC-PUFA for membrane lipid synthesis and structural integrity of the intestinal barrier in the fetus may help protect the immature intestine from an excessive inflammatory response. Further studies are needed to investigate the regulation of ARA and EPA metabolism in relation to intestinal inflammation during early development.
In conclusion, the present study demonstrated that DHA, at a physiological concentration present in human breast milk, significantly attenuated the proinflammatory cytokine response in healthy fetal, NEC, and adult human intestinal epithelial cells after IL-1 $\beta$ challenge. In addition, DHA downregulated exaggerated NFk $\beta 1$ and IL-1R1 gene expression in fetal IEC, which may partly contribute to its observed anti-inflammatory effects. Further, ARA had potent anti-inflammatory effects in healthy fetal IEC, but not in NEC or adult IEC. Thus, data from this study support the notion that DHA may be an important anti-inflammatory nutrient for prevention and treatment of NEC during early human development. Well controlled randomized clinical trials are clearly needed to further investigate the clinical significance and appropriate ratios of DHA, EPA, and ARA supplementation for prevention of NEC in premature infants.

\section{METHODS}

Human fetal and adult IEC in culture were supplemented with 100 $\mu \mathrm{mol} / \mathrm{l} \mathrm{PAL}$ or ARA or EPA or DHA or ARA+DHA for $48 \mathrm{~h}$ in the media containing delipidized fetal bovine serum (FBS). Control IEC were treated with fatty acid-free bovine serum albumin only in the media. PAL, a saturated fatty acid present in human breast milk, was included as a positive control. Fatty acid composition of human IEC in culture was determined $48 \mathrm{~h}$ after fatty acid supplementation. Cytokine concentrations and gene expression in control and fatty acid supplemented IEC were determined 6 and $4 \mathrm{~h}$ after IL-1 $\beta$ challenge, respectively. All experiments were done in triplicates.

\section{Human Fetal and Adult Intestinal Epithelial Cell Lines}

Human fetal intestinal epithelial cells isolated and cultured from a 20 -wk gestation healthy fetal ileum (H4 cells) and a resected ileum of a 25-wk gestation neonate diagnosed with NEC (NEC-IEC) were used as models for human fetal IEC. These cells were prepared after obtaining an informed consent according to the Partners IRB \#1999P003833 at Massachusetts General Hospital. Details on H4 and NEC-IEC in primary culture have been described previously $(4,11,20,24)$. Briefly, H4 cells were cultured in Dulbecco's modified Eagle medium (Life Technologies, Grand Island, NY) supplemented with 10\% heat-inactivated FBS (Life Technologies), 1\% hepes buffer (Life Technologies), $1 \%$ glutamax (Life Technologies), 1\% nonessential amino acids (Life Technologies), $0.2 \mathrm{U} / \mathrm{ml}$ insulin (Eli Lilly and Company, Indianapolis, IN), and $1 \%$ antibiotic-antimycotic (Life Technologies) in a sterile cell culture humidifier at $37^{\circ} \mathrm{C}$ and $5 \% \mathrm{Co}_{2}$. NEC-IEC were cultured in Opti-Minimal Essential Medium (Opti-MEM, Life Technologies) supplemented with $10 \%$ heat-inactivated FBS (Life Technologies), $0.2 \mathrm{U} / \mathrm{ml}$ insulin (Eli Lilly and Company), $20 \mathrm{ng} / \mathrm{ml}$ epidermal growth factor (Life Technologies), and 1\% antibiotic-antimycotic (Life Technologies) in a sterile cell culture humidifier at $32{ }^{\circ} \mathrm{C}$ and $5 \% \mathrm{Co}_{2}$. Adult human colonic cell lines namely, $\mathrm{Caco} 2$ derived from human colonic carcinoma, purchased from American Type Culture Collection (Manassas, VA) and NCM460, a primary cell line derived from normal human colon, purchased from Incell (SA, Texas) were used as models for adult IEC and were cultured as previously published (24). Briefly, $\mathrm{Caco}_{2}$ IEC were cultured in Dulbecco's modified Eagle medium (Life Technologies) supplemented with $10 \%$ heat-inactivated FBS (Life Technologies), 1\% Hepes buffer (Life Technologies), 1\% glutamax (Life Technologies), 1\% nonessential amino acids (Life Technologies) and 1\% antibiotic-antimycotic (Life Technologies), in a sterile cell culture humidifier at $37^{\circ} \mathrm{C}$ and $5 \% \mathrm{CO}_{2}$. The NCM 460 IEC were cultured in M3:Base A (M3A, Incell) media supplemented with $10 \%$ heat-inactivated FBS (Life Technologies), and 1\% antibiotic-antimycotic (Life Technologies), in a sterile cell culture humidifier at $37^{\circ} \mathrm{C}$ and $5 \% \mathrm{Co}_{2}$. 


\section{Fatty Acid Supplementation of Human Fetal and Adult IEC in Culture}

Fatty acids ( $\geq 99 \%$ purity) including PAL, ARA, EPA, and DHA were purchased from Nu-Chek prep (Waterville, $\mathrm{MN}$ ). When human fetal and adult IEC in culture reached $70 \%$ confluence, they were supplemented with $100 \mu \mathrm{mol} / \mathrm{l} \mathrm{PAL}$ (positive control) or ARA or EPA or DHA or ARA+DHA (50 $\mu \mathrm{m}$ each) bound to fatty acid-free bovine serum albumin (Sigma Aldrich, St. Louis, MO) at 3:1 ratio (25) in media containing delipidized FBS (Gemini Bio-Products, Sacramento, CA) for $48 \mathrm{~h}$. Control IEC were treated with fatty acid-free bovine serum albumin only in the media. IEC cell viability in culture after $48 \mathrm{~h}$ of fatty acid supplementation was determined by alamar blue (Life Technologies) and LDH assay (Cayman Chemical, Ann Arbor, MI) according to manufacturer's protocol. Cell viability was not affected by any of the fatty acid supplementation compared to control unsupplemented H4, NEC-IEC, Caco2, or NCM460 IEC in culture (data not shown).

\section{Fatty Acid Analysis of Human IEC in Culture}

Forty-eight hours after fatty acid treatment, culture media was aspirated and IEC in culture were washed three times with phosphate buffered saline ( $\mathrm{pH}$ 7.0) buffer (Sigma Aldrich). Washed IEC cells were harvested by trypsinization and collected in $5 \mathrm{ml}$ deionized distilled water. IEC suspended in sterile, deionized water were centrifuged at $4,000 \mathrm{rpm}$ and supernatant was aspirated after centrifugation; IEC were resuspended in $800 \mu \mathrm{l}$ deionized water and frozen immediately at $-80^{\circ} \mathrm{C}$ for fatty acid analysis.

Fatty acid analysis of human IEC harvested after $48 \mathrm{~h}$ of fatty acid supplementation was done at the laboratory of Dr. JT Brenna (Cornell University, Ithaca, NY), according to the methods described in detail previously $(25,26)$. Enrichment of fatty acids in IEC after supplementation (Table 1) is expressed as a ratio of fatty acids (\% total fatty acids) in supplemented IEC to unsupplemented control IEC.

\section{IL-8 and IL-6 Protein Analysis}

IEC in culture were stimulated with $1 \mathrm{ng} / \mathrm{ml}$ of IL- $1 \beta$ after $48 \mathrm{~h}$ of fatty acid supplementation. Six hours after IL-1 $\beta$ exposure, CFS from IEC in culture were collected, gently vortexed, and frozen immediately in aliquots at $-80^{\circ} \mathrm{C}$ for IL- 8 and IL- 6 analysis. IL- 8 and IL- 6 concentration in CFS was determined by enzyme-linked immunosorbent assay using human IL-8 and IL-6 ELISA kits (R\&D Systems, Minneapolis, $\mathrm{MN}$ ). Protein content of IEC in culture were determined by lysing cells in monolayer with protein lysis buffer (Thermo Scientific, Rockford, IL) and measuring protein content of cell lysate by nanometer. IL-8 and IL- 6 concentration in CFS was expressed per mg protein in cell lysates ( $\mathrm{ng} / \mathrm{mg}$ protein).

\section{Gene Expression Analysis}

The fatty acid effects on gene expression were determined only in fetal H4 IEC (since human NEC-IEC was a primary cell line with limited availability and DHA had consistent effects in H4 and NEC-IEC). Similarly, mRNA expression was measured only in adult Caco 2 cells since fatty acids effects were similar between adult Caco 2 and NCM 460 IEC in culture.

$\mathrm{H} 4$ and Caco2 IEC were exposed to $1 \mathrm{ng} / \mathrm{ml}$ of IL-1 $\beta$ after $48 \mathrm{~h}$ of fatty acid supplementation. Four hours after IL-1 $\beta$ exposure, IL-8, IL-6, IL-1R1, and NFk $\beta 1$ mRNA expression, relative to glyceraldehyde 3-phosphate dehydrogenase (GAPDH) as a house-keeping gene, was determined in IEC by quantitative reverse transcription PCR, as described in detail previously $(4,11)$. Briefly, total RNA from IEC were extracted using the Qiagen RNA kit (Qiagen, Valencia, CA), according to manufacturer's instruction. RNA was reverse transcribed using a Qiagen RNA reverse transcriptase kit (Qiagen), according to manufacturer's instructions. mRNA expression was quantified using Sybergreen master mix system (BioRad, Hercules, CA), following the manufacturer's protocol and as described in detail previously $(4,11)$. Primers for IL-8, IL-6, IL-1R1 NFk $\beta 1$, and GAPDH (house-keeping gene) were synthesized at the MGH genomics core facility. Fatty acid effects on mRNA expression of IL-8, IL-6, IL-1R1, and NFk $\beta 1$ are expressed as a relative ratio of respective mRNA expression in the control (unsupplemented) IEC (arbitrarily set as 1).

\section{Statistical Analysis}

All experiments were done in triplicates. Fatty acid effects on IL-8 (ng/mg protein and mRNA), IL-6 (ng/mg protein and mRNA), and IL-1R1 and NFk $\beta 1$ mRNA were determined by one-way ANOVA and post-hoc Tukey test using Excel 2013 software. Fatty acid fold enrichment (Table 1) and ARA, EPA elongation (Figure 1) data were log transformed for statistical analysis. Data are reported as mean \pm SD.

\section{SUPPLEMENTARY MATERIAL}

Supplementary material is linked to the online version of the paper at http://www.nature.com/pr

\section{ACKNOWLEDGMENTS}

The authors acknowledge Nanda Shanmugam (Mucosal Immunology and Biology Research Center, Massachusetts General Hospital for Children, Boston, MA) for assistance with qRT-PCR work; Maureen Garron and Suzette McCarron (Mucosal Immunology and Biology Research Center, Massachusetts General Hospital for Children, Boston, MA) for administrative support.

\section{AUTHOR CONTRIBUTIONS}

V.W.: Contributed significantly to concept, experimental design, experiments, and manuscript preparation; J.T.B.: Contributed significantly to experimental design, technical approach, and manuscript preparation; D.H.W.: helped with experiments and sample analysis; W.Z.: helped with experiments and sample analysis; D.M.: contributed to experiments; K.G.: contributed to experiments; K.S.D.K.: contributed to experiments; P.R.: contributed to experiments; S.M.I.: contributed to technical approach; W.A.W.: contributed significantly to concept, design, and manuscript preparation.

\section{STATEMENT OF FINANCIAL SUPPORT}

This work was supported by grants from the National Institutes of Health (Bethesda, Maryland) R01-HD059126 (to W.A.W. and Reentry Supplement to V.W.), R01-HD12437, P01 DK033506, P30 DK040561 (to W.A.W.); R01-AT007003 (to J.T.B.).

Disclosure: The authors have no competing interests or financial ties to products.

\section{REFERENCES}

1. Neu J, Walker WA. Necrotizing enterocolitis. N Engl J Med 2011;364: $255-64$.

2. Henry MC, Moss RL. Necrotizing enterocolitis. Annu Rev Med 2009;60:111-24.

3. Gephart SM, McGrath JM, Effken JA, Halpern MD. Necrotizing enterocolitis risk: state of the science. Adv Neonatal Care 2012;12:77-87; quiz 88-9.

4. Nanthakumar N, Meng D, Goldstein AM, et al. The mechanism of excessive intestinal inflammation in necrotizing enterocolitis: an immature innate immune response. PLoS One 2011;6:e17776.

5. Chan KY, Leung KT, Tam YH, et al. Genome-wide expression profiles of necrotizing enterocolitis versus spontaneous intestinal perforation in human intestinal tissues: dysregulation of functional pathways. Ann Surg 2014;260:1128-37.

6. De Plaen IG, Liu SX, Tian R, et al. Inhibition of nuclear factor-kappaB ameliorates bowel injury and prolongs survival in a neonatal rat model of necrotizing enterocolitis. Pediatr Res 2007;61:716-21.

7. Brenna JT, Varamini B, Jensen RG, Diersen-Schade DA, Boettcher JA, Arterburn LM. Docosahexaenoic and arachidonic acid concentrations in human breast milk worldwide. Am J Clin Nutr 2007;85:1457-64

8. Zhang P, Lavoie PM, Lacaze-Masmonteil T, Rhainds M, Marc I. Omega-3 long-chain polyunsaturated fatty acids for extremely preterm infants: a systematic review. Pediatrics 2014;134:120-34.

9. Carlson SE, Montalto MB, Ponder DL, Werkman SH, Korones SB. Lower incidence of necrotizing enterocolitis in infants fed a preterm formula with egg phospholipids. Pediatr Res 1998;44:491-8.

10. Fewtrell MS, Morley R, Abbott RA, et al. Double-blind, randomized trial of long-chain polyunsaturated fatty acid supplementation in formula fed to preterm infants. Pediatrics 2002;110(1 Pt 1):73-82.

11. Ganguli K, Meng D, Rautava S, Lu L, Walker WA, Nanthakumar N. Probiotics prevent necrotizing enterocolitis by modulating enterocyte genes that 
regulate innate immune-mediated inflammation. Am J Physiol Gastrointest Liver Physiol 2013;304:G132-41.

12. Claud EC, Lu L, Anton PM, Savidge T, Walker WA, Cherayil BJ. Developmentally regulated IkappaB expression in intestinal epithelium and susceptibility to flagellin-induced inflammation. Proc Natl Acad Sci USA 2004;101:7404-8.

13. Liu Y, Chen F, Odle J, et al. Fish oil enhances intestinal integrity and inhibits TLR4 and NOD2 signaling pathways in weaned pigs after LPS challenge. J Nutr 2012;142:2017-24.

14. Innis SM, Dai C, Wu X, Buchan AM, Jacobson K. Perinatal lipid nutrition alters early intestinal development and programs the response to experimental colitis in young adult rats. Am J Physiol Gastrointest Liver Physiol 2010;299:G1376-85.

15. Kihara A. Very long-chain fatty acids: elongation, physiology and related disorders. J Biochem 2012;152:387-95.

16. Harkewicz R, Du H, Tong Z, et al. Essential role of ELOVL4 protein in very long chain fatty acid synthesis and retinal function. J Biol Chem 2012;287:11469-80.

17. Ohtsuka Y, Okada K, Yamakawa Y, et al. $\omega$-3 fatty acids attenuate mucosal inflammation in premature rat pups. J Pediatr Surg 2011;46:489-95.

18. Lu J, Jilling T, Li D, Caplan MS. Polyunsaturated fatty acid supplementation alters proinflammatory gene expression and reduces the incidence of necrotizing enterocolitis in a neonatal rat model. Pediatr Res 2007;61:427-32.
19. Garlanda C, Mantovani A. Ligands and receptors of the interleukin-1 family in immunity and disease. Front Immunol 2013;4:396.

20. Nanthakumar NN, Fusunyan RD, Sanderson I, Walker WA. Inflammation in the developing human intestine: A possible pathophysiologic contribution to necrotizing enterocolitis. Proc Natl Acad Sci USA 2000;97:6043-8.

21. Massaro M, Habib A, Lubrano L, et al. The omega-3 fatty acid docosahexaenoate attenuates endothelial cyclooxygenase-2 induction through both NADP $(\mathrm{H})$ oxidase and PKC epsilon inhibition. Proc Natl Acad Sci USA 2006;103:15184-9.

22. Jacobi SK, Moeser AJ, Corl BA, Harrell RJ, Blikslager AT, Odle J. Dietary long-chain PUFA enhance acute repair of ischemia-injured intestine of suckling pigs. J Nutr 2012;142:1266-71.

23. Norris PC, Dennis EA. Omega-3 fatty acids cause dramatic changes in TLR4 and purinergic eicosanoid signaling. Proc Natl Acad Sci USA 2012;109:8517-22.

24. Claud EC, Savidge T, Walker WA. Modulation of human intestinal epithelial cell IL-8 secretion by human milk factors. Pediatr Res 2003;53:419-25.

25. Reardon HT, Hsieh AT, Park WJ, et al. Dietary long-chain polyunsaturated fatty acids upregulate expression of FADS3 transcripts. Prostaglandins Leukot Essent Fatty Acids 2013;88:15-9.

26. Park WJ, Kothapalli KS, Lawrence P, Tyburczy C, Brenna JT. An alternate pathway to long-chain polyunsaturates: the FADS2 gene product Delta8desaturates 20:2n-6 and 20:3n-3. J Lipid Res 2009;50:1195-202. 